\title{
UPON GENERAL INFECTION BY THE BACILLUS PYOCYANEUS IN CHILDREN.
}

\author{
By E. P. Williams, M.D., ${ }^{1}$ and Kenneth Cameron, B.A., M.D.
}

From the Molson Pathological Laboratory, $M^{\prime}$ Gill University, Montreal.

THE observations of Bouchard, Charrin ( ${ }^{1}$ ), Ruffer, and others have shown that the Bacillus pyocyaneus, obtained primarily as a contamination in the pus of wounds, is very definitely pathogenic for certain of the lower animals, notably rabbits. While occasionally met with in association with other microbes, it has been but rarely found as a primary cause of disease in man.

The cases here related being, beyond doubt, of this nature are therefore of more than usual interest.

CAsE 1.-C. E., an illegitimate male child; born on 24th October 1893 , in the Montreal Maternity Hospital, and admitted on November 17th to the Montreal Foundling and Infant Nursery with his mother, a Swedish woman in good health. $\mathrm{He}$ was entirely breast-fed, and gained steadily until the twenty-second week of age, when he became restless and ill, and began to lose weight. These indefinite symptoms, for which no cause could be assigned, continued for 5 weeks, when diarrhœe with green stools set in, accompanied by fever $\left(99^{\circ}-100^{\circ}\right)$, abdominal pain and tenderness, but no tympanites. About the first week of May a group of half a dozen purple papules, 3-7 mm. in diameter, appeared on the abdomen on each side, midway between the umbilicus and the flank. The skin over the abdomen was relaxed, dry, and wrinkled. The child was then in a low, depressed state, lying listlessly in his mother's arms, frequently moaning, especially when disturbed. During the following fortnight numerous papules appeared, extending up and down from the original groups and across the hypogastrium, in the form of an irregular horseshoe; still later many of these became confluent, and others appeared on the abdomen and chest. By the 18th the depression became extreme; the green stools continued, although the abdominal pain and tenderness had ceased. The abdominal facies was very marked. The child could not, or would not, move his limbs. The legs were flexed upon the thighs and the thighs upon the abdomen, and any attempt to straighten them out caused the child to moan; when released they at once returned to their former position. The papules now, for the first time, were seen on the thighs and shoulders.

1 Since this paper was put into type Professor Adami has written to say that Dr. Williams has succumbed to an attack of blood poisoning, contracted during the performance of his pathological work. Dr. Williams was an earnest investigator, and there seems to be little doubt that overwork left him very susceptible to infection. - ED. 
On the 22nd there was a very profuse epistaxis, followed by a refusal to take food. On the 23rd the superficial abdominal veins became distended. On the 25th subcutaneous hæmorrhages occurred from between the toes and from papules on the right thigh and back. Nearly all the spots were now of a darker colour. The next day a slight purulent discharge from the left ear was observed. The child became comatose, and died on 27th May, two months from the onset of the illness. The case was looked upon as one of purpura hæmorrhagica of septic origin.

An autopsy was performed shortly after death. The body was not markedly emaciated, there being a fair quantity of subcutaneous fat. Sections through the blue spots showed deep pigmentation of the skin and infiltration of the subjacent fat and loose tissue with dark blood.

The muscles and the thoracic and abdominal organs, except the spleen, were very pale. The heart was almost empty, save for a little fluid blood in the left auricle. The spleen was firm and of a deep crimson colour, the capsule firmly adherent, and the Malpighian bodies distinet. The kidneys were large, and on the inferior surfaces there were two or three small purplish spots, over one of which the capsule was puckered and thickened. On section these resernbled small, firm infarcts. There was no sign of any marked inflammatory lesion in the intestines, though the mucous membrane was thickened.

Microscopic examination of the kidneys, liver, and spleen, showed that many of the capillaries were blocked with emboli, formed by minute bacilli; in some instances the micro-organisms had passed through the walls of the vessels, infiltrating the surrounding tissue. There was a slight generalised parenchymatous nephritis, the tubules, especially in the convoluted portion, being swollen and cloudy and filled with granular débris. The liver cells were also irregular and cloudy.

Minute portions of tissue were removed from the spleen pulp, and from one of the hæmorrhagic spots on the kidney, and put into culture tubes of gelatine (containing 1 per cent. of Mercks' peptone and 0.5 per cent. of sodium chloride), and of agar-agar ( 1 per cent. peptone, 1 per cent. glycerine, and 0.5 per cent. sodium chloride). These tubes were kept at the ordinary temperature of the room, and on the sixth day minute creamy white striæ or films were seen upon the surface of the media. The tubes were then placed in an incubator, when by the following observations the growth was found to be that of the B. pyocyaneus.

We shall enter at some length into the cultural characteristics of the organism isolated by us, inasmuch as the growths did not wholly correspond with those described by other writers on the $B$. pyocyaneus. To this point we shall refer later.

On agar-agar the growth was moist, spread rapidly along the needle track and radiated over the surface of the medium, which soon became a fluorescent light green, usually with a slight bluish tinge at the edge of the growth. Later, the agar became a deep grass-green, and finally a nut-brown.

Eight weeks later some agar tubes prepared without glycerine were inoculated from the first ones. The growth was at first the same, but after two days the edges became moist, and soon the moisture ran to the bottom of the tube where the growth rapidly continued. Upon the surface of the primary growth minute stellate spots, with a dry metallic lustre appeared, and rapidly coalesced and spread until the entire surface presented the lustre.

On the gelatine a thin greyish film appeared on the surface, while the upper portion of the medium rapidly liquefied. This film became thick and floated on the surface, while colonies sank and continued the liquefaction. The liquefied portion was reddened with a greenish cloud in the upper part near the surface. When the tube was shaken the entire fluid became a bright opalescent bluish-green. This colour faded, but could be observed near the surface after standing. It could be at any time reproduced by shaking. Later 
the gelatine all became liquefied and of a reddish colour, but when shaken turned a grass-green. The growth sank to the bottom of the tube. In an old tube no green could be produced, and the colour became a deep brownish-red.

Cultures made from papules on the skin remained sterile, in consequence, perhaps, of the presence of the bichloride of mercury with which the skin was washed, and others from the contents of the small intestines contained intestinal bacteria but no B. pyocyaneus.

On the tenth day after the first series of cultures had been made, a second series of tubes of beef-broth, and of egg-glycerine were inoculated from the agar and gelatine which had shown the characteristic growth.

In alkaline beef-broth ( 1 per cent. peptone and 0.5 per cent. sodium chloride), tubes placed in the incubators at $37^{\circ} \mathrm{C}$., a greyish film appeared on the surface in 24 hours. On shaking, the entire broth became cloudy and of a bluish-green colour, which later turned to a grass-green. The colour would fade and reappear as in the gelatine.

On egg albumen a creamy bluish-white growth spread rapidly along the needle track. The medium became first a bluish-green, and the liquid formed was of an opalescent or cloudy blue.

Later, the egg was of a deep blue colour, and the liquid was green at the upper part and red at the bottom of the tube. On shaking, the egg dissolved, and all became a dark green. In old tubes the colour was generally a deep greenish-blue.

On egg glycerine (1 per cent. glycerine) the growth resembles that on egg. In 36 hours the medium became a beautiful bluish-green, which in the course of 7 days turned to a deep blue colour, except at the edge of the growth. The liquefied portion was blue and cloudy. In old tubes, when all was liquefied, the colour slowly became red.

On potato, in 15 hours, the growth spread from the needle track over the surface. The growth was moist and fawn-coloured, while the potato became a bright green. Later the growth became dry and shiny, and, like the potato, turned a dark dirty green.

In broth containing 0.7 per cent. boric acid, many long bacilli were seen in active growth, some were curved, others had slightly clubbed ends. No colour was produced.

On beef-broth with 5.5 per cent. peptone, the growth was rapid, the bacilli dividing and forming short chains, while many became from three to six times longer than in the broth with 1 per cent. peptone.

All the cultures after a few days emitted the peculiar odour of trimethylamine, characteristic of cultures of $B$. pyocyaneus.

The blue crystals of pyocyanine were readily obtained by adding chloroform to the alkaline fluid cultures and evaporating slowly to dryness.

If to any of these cultures chloroform was added, it assumed a beautiful Cambridge-blue colour. On rendering the fluid acid by dilute sulphuric acid the blue coloration of the chloroform disappeared, and the supernatant fluid became pink. The addition of ammonia again rendered the chloroform blue.

To inoculate a rabbit, an agar-agar roll tube was prepared, and from it a minute colony was removed and placed in a tube of beef-broth. Twentyfour hours later, when the broth showed a faint greenish colour, $0 \cdot 5$ c.c. was injected into a vein in the ear of a healthy rabbit. In less than 24 hours the animal was unable or unwilling to move, and the hind-legs became stiff.

A slight diarrhoea set in, all the muscles stiffened, and death occurred 40 hours after inoculation. On examination, small punctate red hæmorrhages were seen in the mucous membrane of the stomach. There were none in the intestines or on the skin. 
The urine contained a small quantity of albumen.

Microscopic examination of the organs showed conditions similar to those met with in the organs of the child, though the vessels were somewhat more markedly affected.

Culture tubes prepared from the blood, liver, spleen, kidney, and urine showed in 24 hours the same growth and colour production as those from the child, but of a more pronounced type. The growth was more rapid and the colour more durable.

It was noted that although the original cultures from the rabbit were afterwards exposed to diffused sunlight for about five hours daily for nearly three weeks, their virulence had not diminished.

It is true that our test-tubes were of fair size $(18 \mathrm{~mm}$. in diameter), and that through the growth and colour production the broth was opaque. Nevertheless, bearing in mind the outcome of recent researches, we had not expected to find the strong sunlight of a Montreal summer absolutely without effect upon the virulence of the bacillus.

CASE 2.-C. B., an illegitimate female child ; born in the Montreal Maternity, 8th April 1894, was nursed by its mother and left by her in the nursery on 7 th June. The infant was poorly nourished, small and thin, weighed $7 \mathrm{lb}$. $4 \mathrm{oz}$, and had a purulent discharge from both ears. She was fed on a mixture of Nestlé's Food, peptonised milk and cream. During the first week there was a gain of $4 \mathrm{oz}$, and the discharge from the ears ceased. After that she steadily failed, the stools became frequent, green, and very offensive.

Treatment with lavage and salicylate of bismuth was of no avail. There was a general lividity of a most pronounced type of the whole body, with a dozen or so pustules full of yellow pus on the head, but no purpuric spots or cutaneous hæmorrhages were ever observed.

Two days before death the lividity became extreme, and there was general rigidity of the muscles. The child died on 1st July, three weeks after admission.

Two days before death tubes of beef-broth and gelatine were inoculated with the pus from one of the pustules on the head, and from some blood taken from the tip of a finger. In neither case did the characteristic growth of B. pyocyaneus appear.

At the autopsy, three hours after death, the body was found to be extremely emaciated, little or no subcutaneous fat being present.

All the abdominal and thoracic organs were pale.

The mucous coat of the stomach and intestines was thickened, and presented a few scattered punctate hæmorrhages, especially in the small intestine. The bladder was distended with pale urine.

Culture tubes of gelatine were inoculated from the kidney, spleen, liver, heart blood, and urine, which, with the exception of the two latter, presented pure growths of the $B$. pyocyaneus, the appearance being identical with those seen in Case 1. The heart-blood and the urine remained sterile.

Two weeks later, from the growth from the spleen a roll tube was prepared, and from a colony in it a tube of broth was inoculated.

After two days, 0.5 c.c. was injected into a vein of a rabbit's ear. The animal died 19 hours afterwards, and, at the post-mortem examination, hæmorrhages were seen in all the organs, but were most marked in the stomach (especially at the cardiac end) and kidneys. Cultures taken from the organs presented the green coloration, but much less marked than in those taken from the child.

The cultures from these cases have been compared from day to day with cultures from bouillon tubes of $B$. pyocyaneus brought from Cambridge in 1892. The only difference has been in the intensity of the colour, which, perhaps on account of the greater age, has been less pronounced in the Cambridge growths. 
In connection with these cases two others may be mentioned which occurred in the same ward. These presented very similar symptoms, but the bacillus under discussion was found in only a single part of the body, and in association with other organisms.

CASE 3.-M. T., an illegitimate female child ; born at the Western Hospital on 20th February 1894, was admitted to the nursery on 13th March in good condition, having been nursed by its mother up to that time. After a month of good health it began to waste, and have green motions, diarrhoea, and fever. The skin soon presented marked lividity, and numerous pustules and subcutaneous abscesses developed all over the body, but especially on the head and back. The large majority of these contained pus of a yellowish colour, but in a few it was brown, and from these latter, growths on nutrient media were obtained, which presented all the characteristics of the B. pyocyaneus. The Staphylococeus pyogenes citreus was also isolated. The child died on 24th June, but unfortunately no autopsy was obtained.

Casz 4.-M. R., an illegitimate female child; born at the Western Hospital on 31st May, and admitted to the nursery 20 hours later. Partly wet-nursed, it maintained its weight $(111 \mathrm{oz}$.) for three weeks, until a purulent discharge from both ears was noticed; this was checked in four days. The symptoms which then followed were diarrhœa, wasting, general lividity, slightly elevated temperature $\left(100^{\circ} \mathrm{F}\right.$.), and cold extremities. Three days before death numerous small hæmorrhagic spots of a port-wine colour appeared on the abdomen and back, a large patch over the right scapula, and a few on the head, shoulders, and legs. A large bedsore developed over the sacrum. The lower limbs became flexed and rigid, the child crying when they were straightened. Temperature fell to $95^{\circ} \mathrm{F}$. The child died on July 23rd.

At the autopsy, performed two hours after death, all the organs were found to be very pale, except the spleen, which was large and dark. There were signs of intestinal catarrh, but no hæmorrhages. There were no lesions in any other organs. Intestines full of yellow fæces, bladder empty. Cultures from the various organs remained sterile, except those from the contents of the cæcum, which, on the following day, showed a green colour, and from amongst the intestinal bacteria the $B$. pyocyaneus was isolated.

Three cases have been reported of the discovery of this bacillus in association with symptoms which were in many respects similar to those in the first two cases here reported.

Ehlers ( ${ }^{2}$ reports the following: $A$ brother and sister, respectively 11 and 12 years old, after a prodromal period, suffered from fever, profuse diarrhœea, enlargement of the spleen, mental depression, and prostration. They were thought to have either typhoid fever or cerebro-spinal meningitis.

About the twelfth day an eruption of papules occurred on the surface of the body and limbs, especially anteriorly. These soon became pustular and bullous, like ecthyma, the bullø containing a blue fluid, and ulcers were formed, with their borders hard and pigmented by hæmorrhages.

One of these children died, and from the pustules, spleen, blood, etc., the $B$. pyocyaneus alone was separated.

Neumann $\left({ }^{3}\right.$ ) reports a case of a child 13 days old, which had symptoms of enteritis, with icterus, petechiæ, and hæmorrhages from the mucous surfaces.

He found, after death, ecchymoses into the skin and mucous membrane of the intestines, a swollen spleen, and parenchymatous degeneration of the kidneys. Microscopically, there was an interstitial hepatitis, which was supposed to be due to syphilis. In the liver and spleen were recognised bacilli, which 
had produced no apparent change in the tissues, and from these organs he obtained pure cultures of the $B$. pyocyaneus (B).

Though these three cases seem to be the only ones recorded in which the $B$. pyocyaneus has been found alone, there are numerous instances in which it has occurred in cases of septic infection in association with other micro-organisms.

It will be seen, upon studying the cultural peculiarities of the germ isolated by us, that the results of the cultivation of this bacillus, although closely resembling, for the most part, those described by Gessard $\left({ }^{4}\right)$, occasionally showed variations.

For instance, while the growth on agar containing glycerine corresponds to that of Gessard, the growth on agar without glycerine gave a metallic lustre, which appears to be the same as that obtained by H. C. Ernst ( ${ }^{5}$ ), with the B. pyocyaneus pericarditidis. On beef-broth, containing 5 per cent. peptone, we also found that the bacillus grew to a greater length. This is another point upon which stress is laid by H. C. Ernst in his endeavour to establish a separate species.

From time to time slight variations resembling those of the B. pyocyaneus of P. Ernst $\left({ }^{6}\right)$ have been noted.

From these facts it seems probable that these bacilli are capable of many variations in form and colour production, according to their environment, and that further experiments will prove Gessard to be correct in his opinion that they are but varieties of races of the same bacillus.

Since our attention has been drawn to the presence of the B. pyocyaneus 10 infant bodies have been examined (post-mortem), with the result that in two a general distribution of the B. pyocyaneus has been found, and in one the bacillus was. found present in the alimentary canal only. This fact, taken in connection with the observations of Neumann and Ehlers, shows clearly that the infant organism is susceptible to the invasion of the bacillus.

The discovery of this micro-organism alone, associated with a train of symptoms closely resembling in so many respects the disease, produced experimentally in rabbits by Ruffer $(7)$,- who found present emaciation, diarrhœea, fever, muscular disorders, albuminuria, and hæmorrhages - the first and last being the most prominent in cases running a slow course, symptoms which are by no means uncommon in infants, especially when they are gathered together in institutions,-indicates that the bacillus is distinctly pathogenic at that early age.

We therefore conclude that in these two cases we have obtained a disease due to the growth of the $B$. pyocyaneus, that, in fact, we have here two typical examples of this very rare condition of true pyocyanic disease, or, if the term be admissible, cyano-pyæmia.

How this disease has originated, that is to say, by what channel the bacillus has gained admission into the organism in these two cases, must still, we think, be a matter of uncertainty. 
It is possible that the entry was through some cutaneous lesion, and this would bring these cases into line with those in which the bacillus is found in association with. other septic micro-organisms in wounds. But it must be acknowledged that in none of our instances was there any antecedent cutaneous disturbance recognisable or noted.

As regards this point, while Bouchard ( $\left.{ }^{8}\right)$ has held that intravenous injection is necessary to produce a general infection by the bacillus, Ruffer seems conclusively to prove that rabbits may be infected by subcutaneous injection.

Another point of entry that might be considered is through suppurative disease of the middle ear. That this is a possibility is shown by the observations of Dr. F. R. Blaxall ( ${ }^{9}$ ), who found $B$. pyocyaneus in the ear discharge occurring as a complication in scarlatina.

In our second case the infant, when it entered the nursery, had a purulent discharge from both ears. This ceased before the progressive emaciation characteristic of the pyocyanic disease began to show itself; it is possible, however, that this was the starting-point of the malady.

It must not be left out of account that the alimentary tract may have been the point of invasion. In favour of this view it is to be noticed that in two instances we have discovered the bacillus in the contents of the small intestine; in one of these in association, in the other unassociated, with the general disease; that Booker $\left({ }^{10}\right)$ has discovered the $B$. pyocyaneus in the intestinal tract of a number of children examined with reference to intestinal bacteria; and that during the first few weeks of life the digestive functions of the infant, especially if artificially fed, are but feebly established, and any impairment of these imperfect secretions renders the food taken a most favourable nidus for the production of abnormal fermentation and the growth of bacteria.

In all the cases here reported signs of gastro-intestinal irritation were present for some time before the skin lesions appeared, but whether this was the result of the intoxication by the bacillus, or only favoured its growth, we have no means of determining, for bacteriological examinations of the stools were not made at the time.

A careful bacteriological investigation of all cases of so-called "infantile marasmus," especially when complicated with skin eruptions (whether petechial or pustular), diarrhœa, fever, and muscular disorders, may demonstrate that the distribution of this micro-organism is much more widespread, and much more disastrous in early life than is generally believed. The close resemblance of the symptoms of Case 1 , to those generally described in text-books as purpura hæmorrhagica, suggests a causative relation between the bacillus and that lesion.

We are aware that several species of bacteria have been described in connection with purpura hæmorrhagica; the most that we wish to suggest is, that in children the $B$. pyocyaneus may more frequently 
be the causal agent in the production of this lesion than has been heretofore held.

In conclusion, we wish to express our sincere thanks to Professor Adami for his assistance and advice throughout the study of these cases.

Note.-This paper was completed in August 1894. Unfortunately the copy first sent to the Journal was lost in transit.

[The first copy was announced by letter, but never arrived.-ED.]

\section{REFERENCES.}

1. Charrin . . . . "La Maladie pyocyanique," Paris, 1889.

2. EHukrs . . . . . Hosp.-Tid., Kjobenh., Mai 1890.

3. Neumann, H. . . . Arch. f. Kinderh., Stuttg., 1890, bd. xxi.

4. Gessard . . . . . Ann. de l'Inst. Pasteur, Paris, 1890, p. 88, and 1891, p. 65.

5. ERNst, H. C. . . . Am. Journ. Med. Sc., Phila., 1893, p. 576.

6. ERNst, P. . . . . Ztschr. $f . H y g$., Leipzig, 1888, bd. ii. s. 2.

7. RUFFEr, M. A. . . "Experimental Investigation into the Nature of the Disease produced by the Inoculation of the Bacillus pyocyaneus," London, 1889.

8. Bouchard . . . . "Cours de Pathologie Générale," 1888.

9 Blaxall . . . Brit. Med. Journ., London, 1894, vol. ii. p. 116.

10. Booker, WM. D. . . Trans. Internat. Med. Congress, ninth session, vol. iii. p. 598. 